ITP-UH-07/95

February 1995

\title{
Integrable models of coupled Heisenberg chains
}

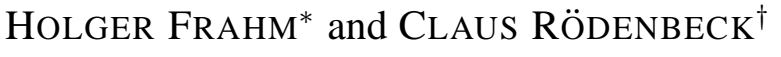 \\ Institut für Theoretische Physik, Universität Hannover \\ D-30167 Hannover, Germany
}

\begin{abstract}
We show that the solutions of the Yang-Baxter equation invariant under the action of the Yangian $Y\left(s l_{2}\right)$ lead to inhomogenous vertex models. Starting from a four dimensional representation of $Y\left(s l_{2}\right)$ we obtain an integrable family of coupled Heisenberg spin- $\frac{1}{2}$ chains. Some thermodynamical properties of this model are studied by means of the algebraic Bethe Ansatz.
\end{abstract}

PACS-numbers: 75.10.Jm 05.30.-d 03.65.-w

*e-mail: frahmeitp.uni-hannover.de

†e-mail: roeden@itp.uni-hannover.de 
Integrable models of one dimensional quantum systems and the related two dimensional classical statistical models have provided much of the insight into correlation effects in low dimensional systems. In particular, Bethe Ansatz methods have been used to study the thermodynamical properties of these systems as well as the excitation spectrum and asymptotic behaviour of correlation functions.

The construction of integrable models within the Quantum Inverse Scattering Method (QISM) is based on solutions of the Quantum Yang Baxter equation [1]

$$
R_{12}(\lambda-\mu) R_{13}(\lambda) R_{23}(\mu)=R_{23}(\mu) R_{13}(\lambda) R_{12}(\lambda-\mu)
$$

The central objects in this approach are the so called $R$-matrices: $R_{i j}(\lambda)$ is a linear operator acting on $V_{i} \otimes V_{j}$ depending on a complex spectral parameter $\lambda$. Hence (1) is an equation on the product space $V_{1} \otimes V_{2} \otimes V_{3}$ with certain finite dimensional vector spaces $V_{i}$. Given a solution of (1) one can construct a family of commuting operators (which include the Hamiltonian) and compute the spectrum using the algebraic Bethe Ansatz.

Particular solutions of Eq.(11) with rational dependence on the spectral parameter are found by looking for $R$-matrices invariant under the action of a simple Lie algebra [2]. In this case $V_{i}$ is to be identified as the representation space of an irreducible finite dimensional representation of this algebra. For the case of $s l_{2}$ this construction leads to the series of higher spin $S U(2)$ Heisenberg chains [3, \#] starting with the $S=1 / 2$ Hamiltonian

$$
\mathcal{H}=\sum_{n} \vec{S}_{n} \cdot \vec{S}_{n+1}-\frac{1}{4}
$$

On the other hand Eq. (11) for given $R_{12}$ can be interpreted as the definition of a quadratic algebra of operators on $V_{3}$. The resulting Hopf algebra is called a Yangian. In the following we shall concentrate on the Yangian associated to $s l_{2}$ which will eventually result in the construction of $S U(2)$-invariant soluble spin chains.

For the simplest so called evaluation representations $V_{m}(a)$ of the Yangian $Y\left(s l_{2}\right)$ the generators $x$ and $J(x)$ of the algebra can be given in terms of irreducible $m+1$-dimensional representations of $s l_{2}$ with generators $\left\{x^{+}, x^{-}, h\right\}$ and an arbitrary complex number $\alpha$ 円

$$
x \in\left\{x^{+}, x^{-}, h\right\}, \quad J(x)=i \alpha x
$$

As was shown by Chari and Pressley every finite dimensional irreducible representation of $Y\left(s l_{2}\right)$ is isomorphic to a tensor product of these evaluation representations [5].

\footnotetext{
${ }^{1}$ For later convenience we rotate the parameter $\alpha$ as compared to the notation used in [5]
} 
Associated to such a representation of the Yangian Drinfel'd has proven that there exists a unique "universal $R$-matrix" which is a rational function of the spectral parameter [6]. Later, Chari and Pressley wrote down explicitly the solution of Eq. (1) associated to a finite-dimensional irreducible representation of $Y\left(s l_{2}\right)$, i.e. $R$-matrices intertwining between two copies of this representation.

In this letter we show that these $R$-matrices can be interpreted as plaquettes containing several vertices of certain inhomogeneous 6 vertex models. This fact allows for the diagonalization of the corresponding transfer matrices using the algebraic Bethe Ansatz. Specifically we study the Hamiltonian (which is integrable by construction) of the quantum spin chain corresponding to the simplest representation of the Yangian beyond the ones given in (3) (which result in the known spin- $m / 2$ Heisenberg models) namely tensor products of two evaluation representations $V_{1}(a) \otimes V_{1}(b)$. It turns out that the resulting model is a system of two $S=1 / 2$ chains with Hamiltonian (2) coupled by two and three spin exchange terms with coupling constants depending on a real parameter.

The approach used in Ref. [5] to compute the solutions of (11) depends on the concept of the intertwiner $I(\alpha-\beta)$ : Given a tensor product of two evaluation representations $V_{m}(\alpha)$ and $V_{n}(\beta)$ the intertwiner is uniquely defined through the following properties: It interchanges the order of the factors, i.e. $V_{n}(\beta) \otimes V_{m}(\alpha) \longrightarrow V_{m}(\alpha) \otimes V_{n}(\beta)$, the Yangian highest weight state $\Omega_{n} \otimes \Omega_{m}$ is mapped onto $\Omega_{m} \otimes \Omega_{n}$ and $I(\alpha-\beta)$ commutes with all elements of the Yangian.

Considered as a representation of $s l_{2} V_{n} \otimes V_{m}$ is isomorphic to the direct sum

$$
V_{n} \otimes V_{m} \cong \bigoplus_{j=0}^{\min \{m, n\}} V_{m+n-2 j} .
$$

By definition the intertwiner commutes with the action of $s l_{2}$. Therefore it can be expanded into a sum of projectors on the irreducible components of the product $V_{m} \otimes V_{n}$ :

$$
I(\alpha-\beta)=\sum_{j=0}^{\min \{m, n\}} c_{j} P_{m+n-2 j},
$$

where $P_{m+n-2 j}: V_{n} \otimes V_{m} \longrightarrow V_{m} \otimes V_{n}$ projects into the spin $(m+n-2 j) / 2$-component of $V_{m} \otimes V_{n}$. The coefficients $c_{j}$ are given by the recursion relation [2]

$$
\frac{c_{j}}{c_{j-1}}=\frac{i \alpha-i \beta+\frac{1}{2}(m+n)-j+1}{i \alpha-i \beta-\frac{1}{2}(m+n)+j-1} .
$$

The $R$-matrix associated to the product $V_{m}(\alpha) \otimes V_{n}(\beta)$ then is given by

$$
R(\lambda)=I(\lambda) \sigma
$$


$\sigma$ being the 'switch map'. These $R$-matrices associated to evaluation representations were first calculated by Kulish et al. [2]].

As shown in Ref. [5] $R$-matrices associated to any tensorproduct of evaluation representations and hence any irreducible representation of $Y\left(s l_{2}\right)$ are uniquely determined by a product of $R$-matrices (7) involving only two factors of the productspace, respectively.

As an example consider the tensor product of four dimensional representations $V_{A}(\lambda)=V_{1}(\lambda) \otimes$ $V_{1}(\lambda+\kappa)$ and $V_{B}(\mu)=V_{1}(\mu) \otimes V_{1}(\mu+\epsilon)$ of $Y\left(s l_{2}\right)$

$$
V_{A}(\lambda) \otimes V_{B}(\mu)=\overbrace{\underbrace{V_{1}(\lambda)}_{1} \otimes \underbrace{V_{1}(\lambda+\kappa)}_{2}}^{A} \otimes \overbrace{\underbrace{V_{1}(\mu)}_{3} \otimes \underbrace{V_{1}(\mu+\epsilon)}_{4}}^{B} .
$$

Following Chari and Pressley the $R$-matrix on this space is given by

$$
R_{A B}(\lambda) \equiv R_{23}(\lambda+\kappa) R_{24}(\lambda+\kappa-\epsilon) R_{13}(\lambda) R_{14}(\lambda-\epsilon)
$$

where $R_{k l}$ is an $R$-matrix of type (7) on the $k$-th and $l$-th factor in $V_{A} \otimes V_{B}$. For the special case of two dimensional ( $\operatorname{spin} \frac{1}{2}$ ) evaluation representations $V_{1}$ it is Yang's R-matrix [7]

$$
R_{k l}(\lambda)=\frac{\lambda-i \sigma_{k l}}{\lambda-i}
$$

$\sigma_{k l}$ interchanges space $k$ and $l$.

In the language of vertex models $R_{k l}(\lambda-\mu)$ can be represented graphically by

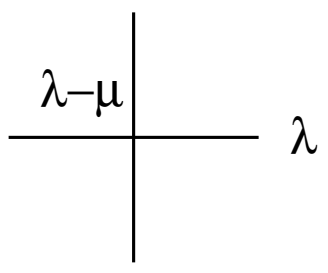

$\mu$

$R(\lambda-\mu)$

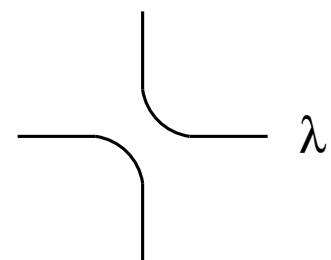

$\lambda$

$\mathrm{R}(0)=\sigma$

where one identifies horizontal lines with the matrix space $k$ and vertical ones with the quantum space $l$. In this framework the $R$-matrix (9) is just a group of vertices in an inhomogeneous vertex model (Fig. 1).

Multiplying in matrix space $N$ of these objects acting on different copies of the quantum space gives the monodromy matrix $\mathcal{T}_{A B}(\lambda-\mu)$. By construction $\mathcal{T}_{A B}$ satisfies the Yang-Baxter equation

$$
R_{A B}(\lambda-\mu)\left(\mathcal{T}_{A B}(\lambda) \otimes 1\right)\left(1 \otimes \mathcal{T}_{A B}(\mu)\right)=\left(1 \otimes \mathcal{T}_{A B}(\mu)\right)\left(\mathcal{T}_{A B}(\lambda) \otimes 1\right) R_{A B}(\lambda-\mu)
$$


As a direct consequence the transfer matrix $T_{A B}(\lambda)=\operatorname{tr} \mathcal{T}_{A B}(\lambda)$ commutes for different values of the spectral parameter $\lambda$. Here the trace is taken with respect to the matrix space. Therefore $T_{A B}(\lambda)$ can be interpreted as a generating functional of commuting operators in the quantum space.

Obviously, $\mathcal{T}_{A B}$ can be written as (see also Fig. 2)

$$
\mathcal{T}_{A B}(\lambda)=\tau(\lambda+\kappa) \otimes \tau(\lambda)
$$

where $\tau(\lambda)$ is the monodromy matrix of the inhomogeneous vertex model constructed from $R_{1 B}(\lambda) \equiv$ $R_{13}(\lambda) R_{14}(\lambda-\epsilon)$ (see ( 8$)$ for the numbering of the different spaces). From (12) we obtain the following relation between $T_{A B}$ and the transfer matrix $t(\lambda)=\operatorname{tr} \tau(\lambda)$

$$
T_{A B}(\lambda)=t(\lambda+\kappa) t(\lambda)
$$

Since $R_{1 B}$ itself is a solution of a Yang-Baxter equation (11) we have $[t(\lambda), t(\mu)]=0$. Consequently the diagonalization of $T_{A B}$ is equivalent to the solution of the eigenvalue problem for $t(\lambda)$.

The latter is obtained in the framework of the algebraic Bethe Ansatz (see e.g. [8]). Starting from the ferromagnetic vacuum $|\Omega\rangle=|\uparrow \uparrow \ldots \uparrow\rangle$ which is an eigenstate of $t_{A B}(\lambda)$ with eigenvalue

$$
\widetilde{\Lambda}_{0}(\lambda)=1+\left(\frac{\lambda}{\lambda+i}\right)^{N}\left(\frac{\lambda-\epsilon}{\lambda-\epsilon+i}\right)^{N}
$$

one constructs eigenstates of $T_{A B}(\lambda)$ with $M$ overturned spins parametrized by $M$ rapidities $\lambda_{j}$ with eigenvalues

$$
\Lambda(\lambda)=\widetilde{\Lambda}(\lambda) \widetilde{\Lambda}(\lambda+\kappa)
$$

Here $\tilde{\Lambda}$ are the eigenvalues of $t(\lambda)$

$$
\widetilde{\Lambda}(\lambda)=\prod_{j=1}^{l} \frac{\lambda_{j}-\lambda+\frac{i}{2}}{\lambda_{j}-\lambda-\frac{i}{2}}+\left(\frac{\lambda}{\lambda+i}\right)^{N}\left(\frac{\lambda-\epsilon}{\lambda-\epsilon+i}\right)^{N} \prod_{j=1}^{l} \frac{\lambda-\lambda_{j}+\frac{3}{2} i}{\lambda-\lambda_{j}+\frac{i}{2}}
$$

and the $\lambda_{j}$ are solutions of the Bethe Ansatz equations

$$
\left(\frac{\lambda_{j}+\frac{i}{2}}{\lambda_{j}-\frac{i}{2}}\right)^{N}\left(\frac{\lambda_{j}-\epsilon+\frac{i}{2}}{\lambda_{j}-\epsilon-\frac{i}{2}}\right)^{N}=\prod_{j \neq k} \frac{\lambda_{j}-\lambda_{k}+i}{\lambda_{j}-\lambda_{k}-i} .
$$

For the construction of a Hamiltonian with local interactions it is necessary that $T_{A B}(\lambda)$ has a shift point, i.e. a value of the spectral parameter $\lambda$, where it degenerates to the shift operator. As shown in Fig. 目 for $\lambda=0$ configurations in quantum space are shifted with an additional coupling between pairs of neighbouring spins. Hence the necessary condition for the existence of a shift point is that this coupling becomes the identity. 
As a direct consequence of the Yang-Baxter equation (11) the $R$-matrices (in the normalization used here) satisfy the unitarity condition

$$
R(\lambda) \sigma R(-\lambda) \sigma=1
$$

Consequently, choosing $\epsilon=\kappa$ we obtain a one-parameter family of monodromy matrices allowing for the construction of Hamiltonians with local interactions.

The Hamiltonian of the integrable spin chain is obtained by taking the logarithmic derivative of the transfer matrix $T_{A B}(\lambda)$ at the shiftpoint $\lambda=0$ :

$$
\left.\frac{i}{2} \frac{d}{d \lambda} \ln T_{A B}(\lambda)\right|_{\lambda=0} \equiv \mathcal{H}(\kappa) .
$$

The resulting spin chain Hamiltonian is $\mathcal{H}(\kappa) \equiv \sum_{n=1}^{N} \mathcal{H}_{n}(\kappa)$ with

$$
\begin{aligned}
\mathcal{H}_{n}(\kappa) \equiv & J_{1}\left(\vec{S}_{2 n-1} \vec{S}_{2 n}+2 \vec{S}_{2 n} \vec{S}_{2 n+1}+\vec{S}_{2 n+1} \vec{S}_{2 n+2}-1\right)+ \\
& +J_{2}\left(\vec{S}_{2 n}\left(\vec{S}_{2 n+2} \times \vec{S}_{2 n+1}\right)+\vec{S}_{2 n-1}\left(\vec{S}_{2 n} \times \vec{S}_{2 n+1}\right)\right)+ \\
& +J_{3}\left(\vec{S}_{2 n} \vec{S}_{2 n+2}+\vec{S}_{2 n-1} \vec{S}_{2 n+1}-\frac{1}{2}\right)
\end{aligned}
$$

The exchange couplings $J_{i}$ depend on the parameter $\kappa$ in the following way

$$
J_{1}=\frac{1}{1+\kappa^{2}}, \quad J_{2}=\frac{2 \kappa}{1+\kappa^{2}}, \quad J_{3}=\frac{\kappa^{2}}{1+\kappa^{2}} .
$$

For $\kappa=0 \mathcal{H}$ reduces to the Hamiltonian (2) of a spin $\frac{1}{2}$ Heisenberg model on a chain of length $L=2 N$, for $\kappa \rightarrow \infty$ one obtains two decoupled systems of length $L=N$ each. For intermediate values of the coupling $\kappa$ one has a system of two coupled Heisenberg chains (see Fig. 3).

From (15) the eigenvalues of (20) are obtained as

$$
E\left(\left\{\lambda_{j}\right\}\right)=\left.\frac{i}{2} \frac{\partial}{\partial \lambda} \ln \Lambda(\lambda)\right|_{\lambda=0}=\sum_{j}\left(\tilde{\epsilon}^{(0)}\left(\lambda_{j}\right)+\tilde{\epsilon}^{(0)}\left(\lambda_{j}-\kappa\right)\right), \quad \tilde{\epsilon}^{(0)}\left(\lambda_{j}\right)=-\frac{1}{2} \frac{1}{\lambda_{j}^{2}+\frac{1}{4}} .
$$

In the thermodynamic limit the solutions of the Bethe Ansatz equations (17) are known to be arranged in bound states if uniformly spaced sets of complex $\lambda_{j}$, so called strings:

$$
\lambda_{j}^{(m)}=x+i \mu_{j}, \quad \mu_{j}=\frac{-m+1}{2}, \frac{-m+3}{2}, \ldots, \frac{m-1}{2} .
$$

As in the Heisenberg model the ground state (in absence of a magnetic field) is made up of $N$ real $\lambda$ 's only. Their densities $\rho(\lambda)$ can be given in terms of a linear integral equation resulting in

$$
\rho(\lambda)=\tilde{\rho}(\lambda)+\tilde{\rho}(\lambda-\kappa), \quad \text { with } \tilde{\rho}=\frac{1}{2 \cosh (\pi \lambda)} .
$$


From (22) the ground state energy $E_{0}$ per spin is given by

$$
\begin{aligned}
\frac{E_{0}}{2 N} & =\frac{1}{2} \int_{-\infty}^{+\infty} d \lambda \rho(\lambda) \epsilon^{(0)}(\lambda)=\int_{-\infty}^{+\infty} d \lambda\left(\tilde{\rho}(\lambda) \tilde{\epsilon}^{(0)}(\lambda)+\tilde{\rho}(\lambda-\kappa) \tilde{\epsilon}^{(0)}(\lambda)\right) \\
& =\ln 2+\frac{1}{2}(\beta(1-i \kappa)+\beta(1+i \kappa)) .
\end{aligned}
$$

The term containing $\beta$-functions

$$
\beta(x)=\frac{1}{2}\left(\psi\left(\frac{x+1}{2}\right)-\psi\left(\frac{x}{2}\right)\right)
$$

( $\psi(x)$ being the digamma function) decreases from $\ln 2$ to 0 as $\kappa$ varies between 0 and $\infty$.

As in the Heisenberg model the low lying excitations above the ground state are made up of an even number of spin-1/2 objects [9] called spinons. These elementary excitations are holes in the distribution of 1-strings parametrized by a rapidity $\lambda$. Their dispersion is found to be

$$
\begin{array}{rlrl}
\epsilon(\lambda) & =\tilde{\epsilon}(\lambda)+\tilde{\epsilon}(\lambda-\kappa), & & \tilde{\epsilon}(\lambda)=\frac{\pi}{2 \cosh (\lambda)} \\
k(\lambda)=\tilde{k}(\lambda)+\tilde{k}(\lambda-\kappa), & \tilde{k}(\lambda)=\arctan (\lambda)-\frac{\pi}{2} .
\end{array}
$$

In Fig. 7 the spinon dispersion $\epsilon(k)$ is shown for several values of the parameter $\kappa$.

Several extensions to the system discussed in this paper are possible:

First, the above methods are easily extended to representations $V_{m}(a) \otimes V_{n}(b)$ of $Y\left(s l_{2}\right)$. This leads to one parametric families of integrable quantum spin chains with alternating spin $S=m / 2$ and $S=n / 2$. In fact, the case of $m=1, n=2$ is the isotropic limit of the alternating spin model constructed by de Vega and Woynarovich [10] at its conformal pointt](for further studies of this model and its generalization to $m>2$ at coupling corresponding to $\kappa=0$ see also Refs. [11, [1] ). In that work the factors in (9) are chosen to be the $R$-matrices arising in the context of the higher spin XXZ models. Again unitarity relation (18) is used to construct families of commuting transfer matrices (see also [13]). While the Yangian approach is limited to $R$-matrices with rational dependence on the spectral parameter an extension to hyperbolic $R$-matrices using the approach of Refs. [13, 10] is straightforward.

Second, irreducible representations of $Y\left(s l_{2}\right)$ of the type $V_{m_{1}}\left(a_{1}\right) \otimes \ldots \otimes V_{m_{k}}\left(a_{k}\right)$ built from more than two evaluation representations (3) can be considered to construct integrable models of $k$ coupled spin chains. While additional multi-spin coupling terms are likely to arise within this construction we

\footnotetext{
${ }^{2}$ In Ref. [10] two commuting Hamiltonian operators $\bar{H}$ and $\tilde{H}$ are constructed for each value of $\kappa$. The method used here produces the rotational invariant vertex model with $\mathcal{H}=\bar{H}+\tilde{H}$.
} 
expect $k-1$ free parameters ( terms from the Hamiltonian.

Finally, the construction of integrable Hamiltonians with local interactions from inhomogeneous vertex models such as the one given in Fig. 1 allows for the generalization to exchange symmetries other than $s l_{2}$ including supersymmetric extensions leading to $t$ - $J$ and extended Hubbard models on coupled chains.

This work has been supported in part by the Deutsche Forschungsgemeinschaft under Grant No. Fr 737/2-1.

\section{References}

[1] L. Faddeev, Sov. Sci. Rev. C 1, 107 (1980).

[2] P. P. Kulish, N. Yu. Reshetikhin, and E. K. Sklyanin, Lett. Math. Phys. 5, 393 (1981).

[3] J. Babujian, Phys. Lett. A 90, 479 (1982).

[4] L. Takhtajan, Phys. Lett. A 87, 479 (1982).

[5] V. Chari and A. Pressley, L'Enseignement Math. 36, 267 (1990).

[6] V. G. Drinfel'd, Soviet Math. Dokl. 32, 254 (1985).

[7] C. N. Yang, Phys. Rev. Lett. 19, 1312 (1967).

[8] L. D. Faddeev and L. A. Takhtajan, J. Sov. Math. 24, 241 (1984), [Zap. Nauch. Semin. LOMI 109, 134 (1981)].

[9] L. D. Faddeev and L. A. Takhtajan, Phys. Lett. A 85, 375 (1981).

[10] H. J. de Vega and F. Woynarovich, J. Phys. A 25, 4499 (1992).

[11] S. R. Aladim and M. J. Martins, J. Phys. A 26, L529 (1993).

[12] H. J. de Vega, L. Mezincescu, and R. I. Nepomechie, Phys. Rev. B 49, 13223 (1994).

[13] N. Andrei and H. Johannesson, Phys. Lett. A 100, 108 (1984). 


\section{Figures}

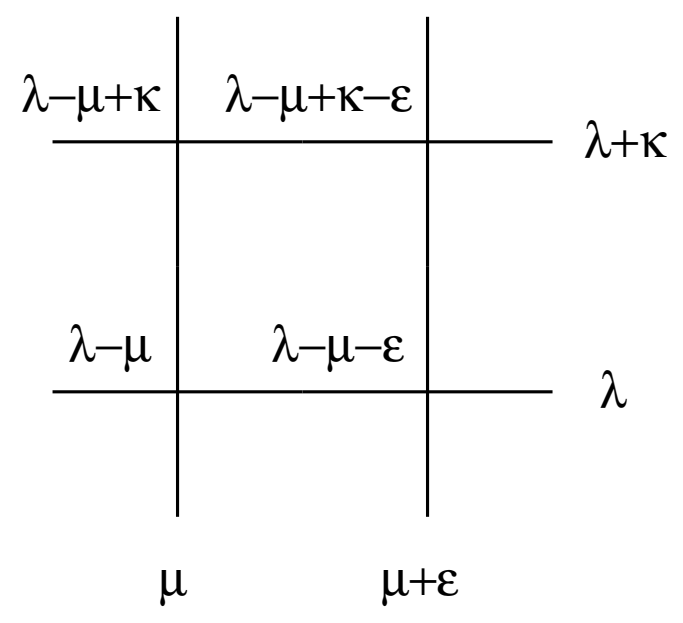

Figure 1: Plaquette of elementary vertices in the $R$-matrix $R_{A B}(\lambda-\mu)$ (9)
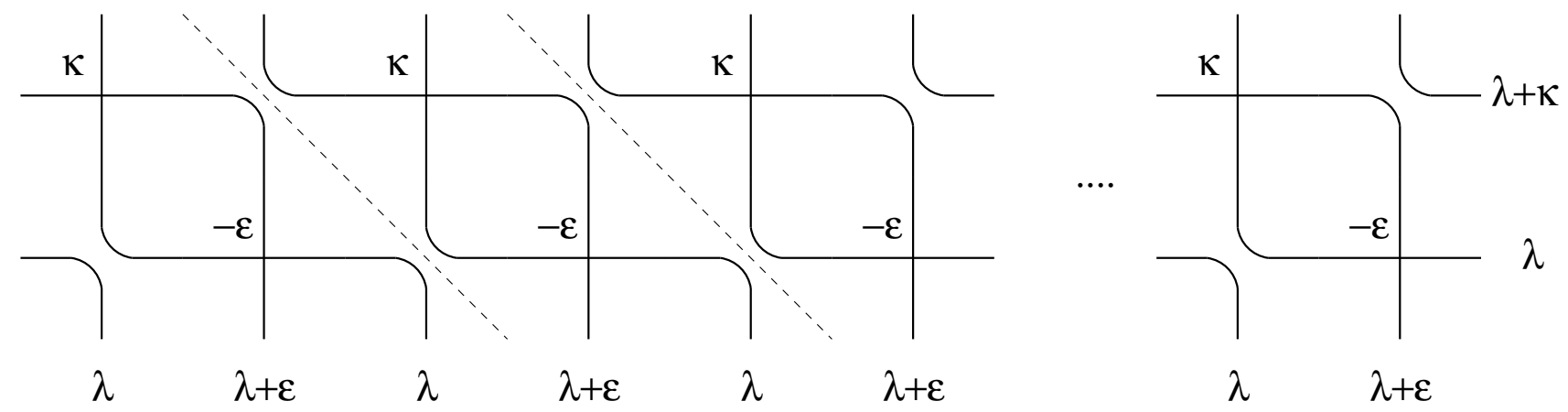

Figure 2: Graphical representation of $\mathcal{T}_{A B}(\lambda)$. 


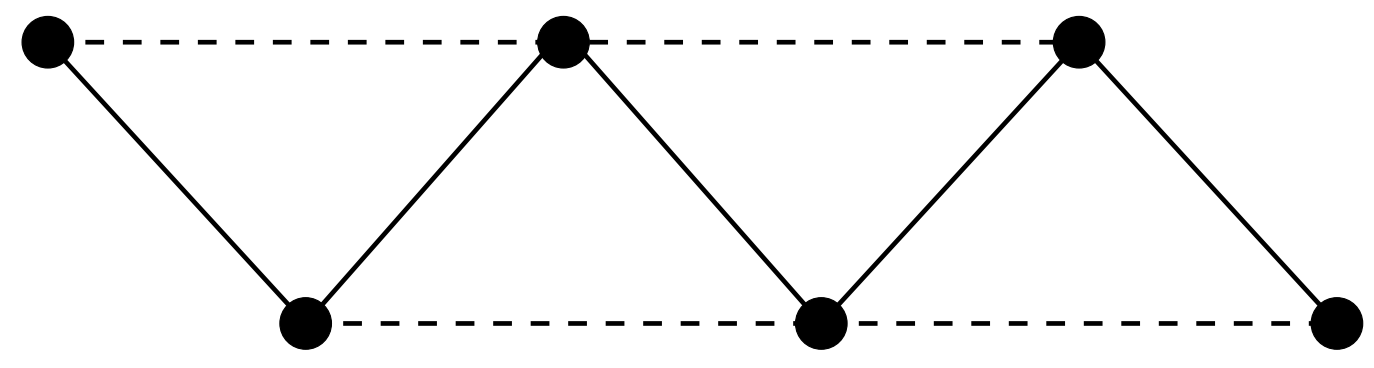

Figure 3: Lattice on which the spin Hamiltonian (20) is defined: The two-spin exchange coupling is $2 J_{1}$ and $J_{3}$ on full and dashed lines, respectively. The three spin exchange $\propto J_{2}$ couples the spins on the corners of each triangle.

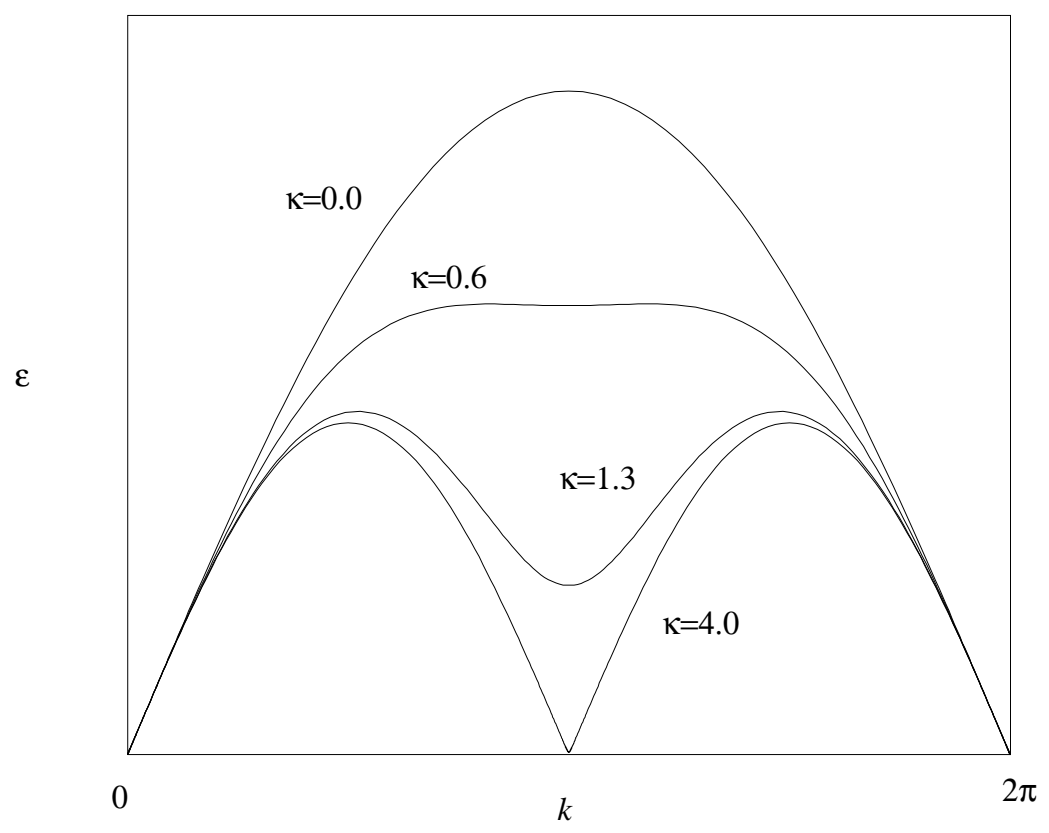

Figure 4: Spinon dispersion for several values of $\kappa$ 